\title{
The structure design and performance analysis of a racing welded steering knuckle
}

\author{
Jing Chen*, Xinyi Shen, Zhicheng Zeng \\ Wuhan University of Technology, Wuhan, China \\ *Corresponding author: 1786953080@qq.com
}

Keywords: steering knuckle; weld analysis; finite element analysis

\begin{abstract}
Steering knuckle as an important safety components of automobiles, in the process of lightweight design, if not taking into account the structure, process and other factors, will inevitably cause the steering knuckle strength and stiffness and other performance reduction, forming a major safety hazard. Based on a four-wheel drive off-road racing car, this paper performs strength analysis and modal analysis of the steering joint after weld analysis, uses multi-body dynamics to break down the hard spot load on the steering joint, and uses the test site test analysis and verification, so as to obtain a good performance steering knuckle.
\end{abstract}

\section{Introduction}

Usually the steering knuckle of passenger cars is mostly made of cast iron with greater strength or a new type of aluminum alloy. However, considering the processing cost and the time cost of production cycle and update iteration, this paper innovatively proposes a welding steering knuckle and studies its structural design and performance. Through the analysis of typical extreme operating conditions, the hard point load of steering knuckle is obtained by using multi-body dynamic model, which is used as a basis for the static strength of the structurally designed steering knuckle in finite meta-software [1].

At the same time, considering that the uneven thermal input in the welding process makes the steel plate produce residual stress and deformation, and the welding order is an important factor affecting welding stress and deformation. In order to improve the assembly accuracy and reduce the change of chassis parameters in the actual movement process, the steering joint is required to have high strength and stiffness in extreme operating conditions, and it is also necessary to study the welding process.

\section{Modeling design}

The steering knuckle is an important component in the car, connecting the hub, control arm and brake assembly. At the same time, in order to coordinate the steering function of the racing car, the steering knuckle is also connected with the arm of the steering pull rod. The steering knuckle also carries the front load of the car, so the force of steering knuckle is large and complex.

According to the overall design of the vehicle to determine the suspension and steering geometry of some parameters. All the connecting parts are connected with ball pins. The fixed position of the caliper requires comprehensive consideration of the relative position of the brake disc, hub and caliper to obtain the maximum braking force. For structural reasons, there is a difference between the form of a racing car's steering tie rod and that of a passenger car. As a result, the steering arm is also structured in a different form than the passenger car. In order to match the steering tie rod, the steering arm needs to be made into two layers, the middle through hole to secure the plug bolt. However, in actual use, only two steel plates are used to fix the plug bolt and the steering tie rod, which can not meet the strength requirements of the use. Therefore, a rib plate is needed to be added in the middle to limit the shaking of the upper and lower steel plates in actual motion, and to increase the strength of the steering knuckle arm under axial force. 
Through theoretical design and related raw materials procurement research, the thickness of the steel plate was initially set at $4 \mathrm{~mm}$. The use of materials is 4130 steel plate. Welding method is carbon dioxide protection welding. Figure 1 below is based on a model in CATIA based on hard point coordinates.

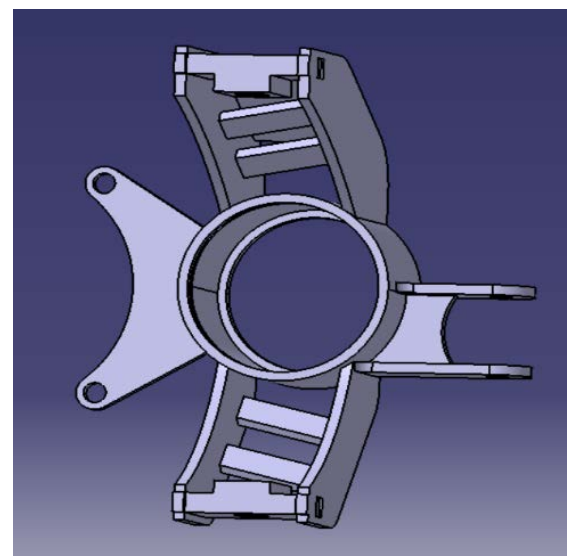

Figure 1.3D model of the steering knuckle is established

\section{Welding analysis}

Most of the welds in this weld are T-joints, which are not symmetrical compared to other welding components, and have more complex temperature and stress strain field. Therefore, in order to ensure the quality of welding under the fewer experiments, the stress strain field of T-joints is simulated in three-dimensional dynamics using finite meta-software SYSWELD, and the temperature field and stress field are simulated at the same time for analysis, which provides a theoretical basis for the optimization of welding process.

SYSWELD is one of the most typical representatives of welding-related CAE software, which enables the corresponding welding calculations, such as mechanical, metal metallurgy, and thermal conduction calculations. According to the characteristics of T-joints, this paper uses finite metaSYSWELD software to perform corresponding numerical simulation.

\subsection{Mathematical model building}

Assuming that there is no flowing material in the solid material, the heat dissipation of the melting pool to the master material can be carried out according to the thermal conduction method, and the control equation of the thermal conduction is

$$
\begin{gathered}
\rho c \frac{\partial T}{\partial t}=\frac{\partial}{\partial x}\left(\lambda \frac{\partial T}{\partial x}\right)+ \\
\frac{\partial}{\partial y}\left(\lambda \frac{\partial T}{\partial y}\right)+\frac{\partial}{\partial z}\left(\lambda \frac{\partial T}{\partial z}\right)+\bar{Q}
\end{gathered}
$$

$\rho$ is the density of the material, $\mathrm{c}$ is the thermal capacity of the material, radon is the thermal conductivity of the material, $\rho, \mathrm{c}$, and the three quantities are all functions of temperature $\mathrm{T}$, and $\mathrm{Q}$ is the strength of the internal heat source[4].

\subsection{The finite meta-model is established}

In this paper, 4130 steel plate as the master material, the hem arm connection point and the side plate welding T-joint as the research object, and the MIG welding method for the corresponding research and analysis.

Because many factors can affect the accuracy of simulation results, simulation simulation calculations are first set for meshing and time steps. It is better to have a fine mesh division near the weld, and the part farther away from the weld can be sparse, so the contradiction between calculation accuracy and calculation speed can be solved by adjusting the mesh division. The cell mesh area at the weld is determined to be $0.3 \mathrm{~mm}$, and the nearby mesh is divided into MAP forms, which are divided by a coarser transition in other places. The following illustration is a divided finite cell grid 
model. Figure 2 has 66,913 units and 72,688 nodes. The properties of the material are determined by defining the substrate part, the weld part, and the heat exchange portion with the air. Finally determine the clamping conditions, ensuring the movement of the components on the Z-axis, in the actual operation process through our purchase of welding fixtures to achieve.

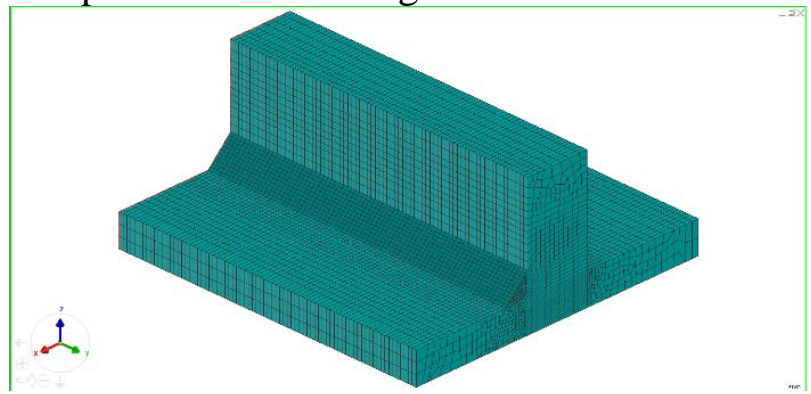

Figure 2. Finite metagrid model

The rational selection of heat source model is the key to simulation calculation. In view of the characteristics of MIG welding, the heating area can be regarded as a double semi-ellipsoid. In Figure 3 below, by measuring the size of the melting pool, the thermal source model in the SYSWELD software is modified, and the welding temperature field can be simulated more accurately. The thermal source model obtained from the simulation of the thermal source nuclear proof tool in SYSWELD is shown in Figure 4.

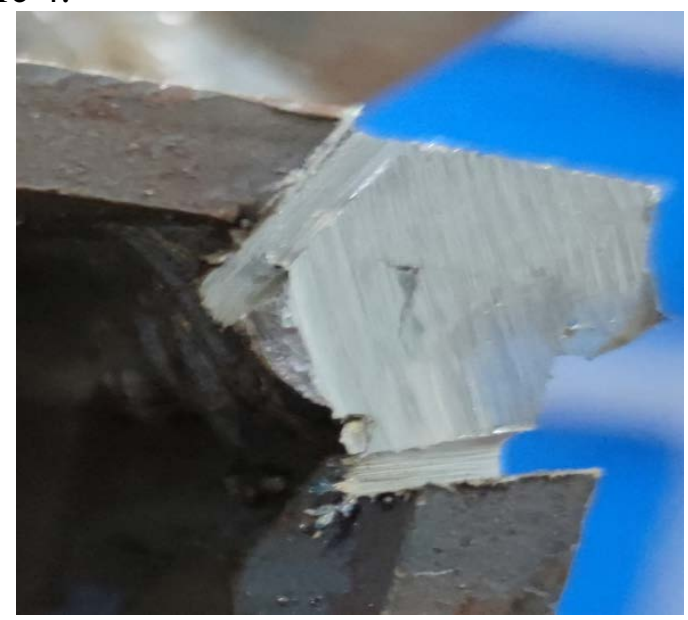

Figure 3. Melting pool shape

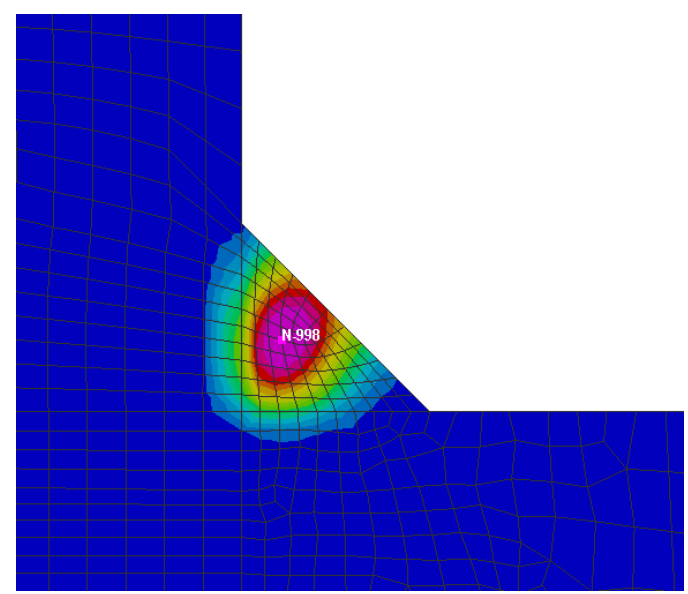

Figure 4. Heat source model

\subsection{Stress strain field analysis}

During welding, the stress field changes over time, and the thermal impact area near the weld is the maximum position of residual stress. As shown in Figure 5, the horizontal and vertical residual average stress center areas distributed on the weld centerline are shown as pull stress. The horizontal 
pull stress of the weld centerline is gradually reduced from the center to the sides to 0 , and then converted to a pressure stress.

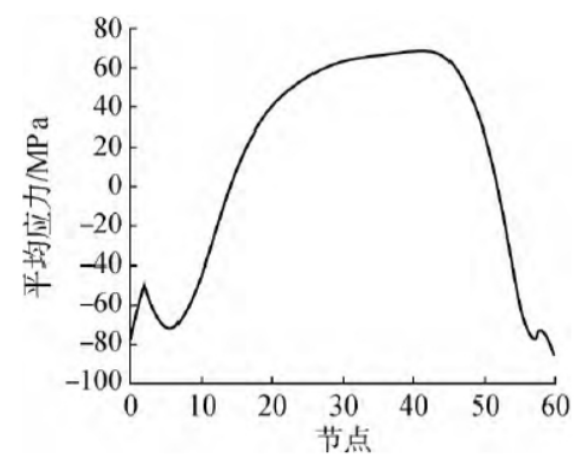

Figure 5. Distribution of horizontal residual average stress

\section{Load acquisition}

\subsection{Load acquisition method}

So far, the static strength of automotive components load decomposition and acquisition has not yet developed a unified industry standard [5]. In this paper, the load of each connection point of the steering knuckle is obtained by using the calculated tire ground force as the simulation input condition of the vehicle multi-body dynamics model.

This method combines traditional dynamic load coefficient calculation with modern multi-body simulation technology, which provides more accurate load boundary conditions for finite factor analysis and improves computational efficiency and accuracy.

\subsection{Build a multi-body dynamics model}

After determining the hard point coordinates of each system according to the race rules and theory calculation, the multi-body dynamic model of the racing car is established in ADAMS/Car according to the assembly relationship.

Due to the large number of parts and components of the racing car, it is difficult to model all parts of the whole vehicle. Since the main purpose of this paper is to obtain the load distribution at the connection points of the steering knuckle, some parts that have little impact on the analysis can be omitted.

The front suspension uses a two-cross-arm independent suspension, which models the double-arm swing arm and the shock converter spring according to the design parameters. The shock absorber is abstracted as the damping spring connecting the lower arm of the body. The steering system is also included in the front suspension, which includes steering, steering lever, steering knuckle, etc. After the subsystems of each model are built, the subsystems are connected through the communicator to form the front suspension assembly model subsystem.

Because the lateral tilt stiffness of the rear suspension meets the design requirements, it is not designed to increase the lateral stability bar to increase the tilt stiffness of the suspension. In the design process of the rear suspension, the subsystems involved include the suspension guide mechanism, shock absorber spring, column hub and half shaft. After the model of each subsystem is established, the assembly is carried out through the communication system. And the process of setting the suspension parameters is the same as that of the front suspension. Next, the engine and transmission system models of the body and frame are successively established, and each subsystem of the Vehicle is connected through the communicator. Each subsystem is found in the database and assembled in the Full-Vehicle Assembly. The resulting racing vehicle assembly model is shown in Figure 6. 


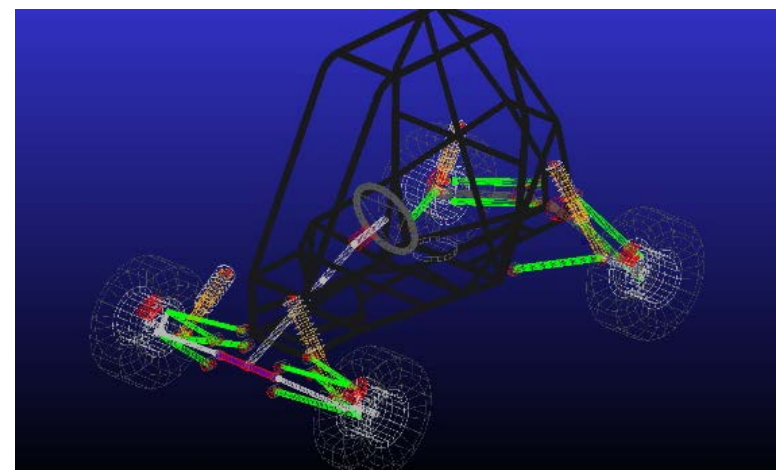

Figure 6. Multi-body dynamics model of the vehicle

\subsection{Typical limit operating conditions analysis and steering knuckle load analysis}

The working conditions of the vehicle in the actual motion engineering are very complex, so in the parts research and development project, it is usually necessary to analyze its strength according to its extreme motion state. Therefore, through investigation and reference to a large number of literature and data, this paper selects the complex working conditions that have a greater impact on the strength of steering knuckle in the working conditions of racing cars to analyze.

An emergency turning condition in which the vehicle turns at maximum lateral acceleration by turning under pit conditions (right). The maximum lateral acceleration that the body structure can provide is $1.7 \mathrm{~g}$. The steering knuckle is subjected to a large longitudinal force, a vertical force and the pull of the steering rod, resulting in a large tilt angle for the vehicle and a very large lateral force on one side of the tire. Over-pit conditions are vehicle impact conditions, steering knuckle are subjected to vertical and vertical impact forces at the same time there is suspension drag.

By converting the tire ground force in this complex operating condition into an input condition for optimizing the simulation, the load of the steering joint is calculated as follows Table 1:

Table 1. Steering load-saving distribution

\begin{tabular}{|c|c|c|c|}
\hline & $\begin{array}{c}\text { X-directional } \\
\text { force division /N }\end{array}$ & $\begin{array}{c}\text { Y-directional } \\
\text { force division /N }\end{array}$ & $\begin{array}{c}\text { Z-directional } \\
\text { force division } \\
/ \mathbf{N}\end{array}$ \\
\hline The arm connection point & -626 & 0 & 1140 \\
\hline Hem arm connection point & 203.57 & 1720.9 & 363.93 \\
\hline The upper swing arm connection point & 397.44 & -3087.4 & -1708.5 \\
\hline Caliper connection point & 0 & -500 & 300 \\
\hline
\end{tabular}

\section{Finite element analysis}

Experience has shown that if the structure of the component can withstand a large peak load without cracks, then its fatigue strength can generally meet the requirements. Therefore, according to the product development needs, this paper conducts static strength and modal analysis of the steering knuckle.

\subsection{Static intensity analysis}

The material of the steering knuckle is 4130 structural steel, the plastic performance is good. The main form of destruction is the yield fails. According to the strength conditions of the third strength theory: $\delta 1-\delta 3 \leq[\delta](\delta 1$ and $\delta 3$ are the first and third principal stresses respectively), the design needs to ensure that the maximum equivalent stress $\delta \mathrm{v}$ of each particle meets the condition when the steering knuckle is loaded:

$$
\delta \mathrm{v} \leq \delta \mathrm{s}=785 \mathrm{MPa}
$$

Import the steering knuckle model into the ANSYS Workbench platform, define the relevant material properties of the 4130 steel plates and mesh them, taking into account the accuracy and 
complexity of the calculation, and the mesh control strategy is: Physics Preference: Mechanical; Relevance:75; Size:1mm; Smoothing: High. The number of tetrahedral grid nodes is 436,855, the number of units is 117,156 , the vast majority of mesh quality is above 0.8 .

The rigid unit (RBE2) is used to simulate the connection between bushings, ball hinges, and components around the steering joint. Fixed Support is applied to the bearing assembly position, and the caliper fixing holes, the upper and lower swing arm fixing holes, and the steering lever fixing holes apply corresponding forces (Force). After the constraint is set, it is solved and the iso-effect force cloud figure 7 is obtained under the typical compound operating conditions of the steering knuckle.

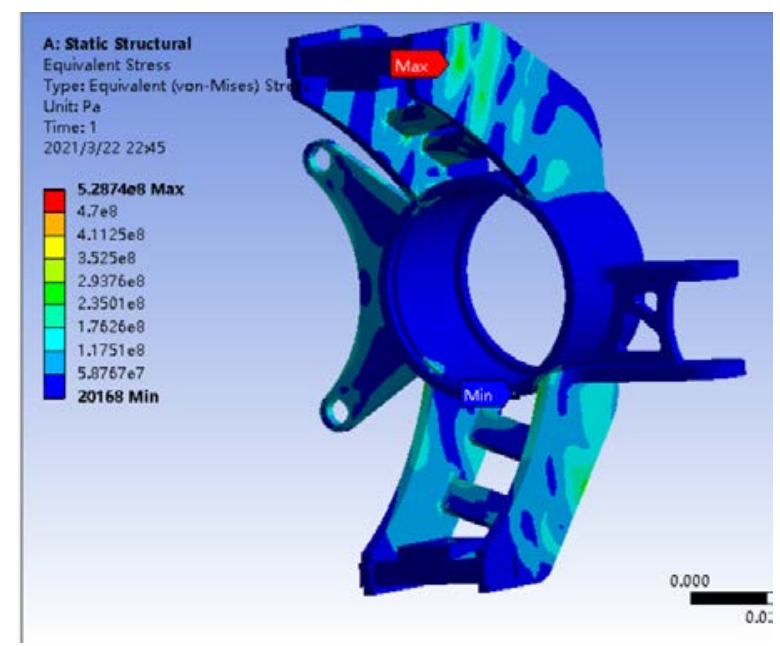

Figure 7. Cloud of effects such as steering knuckle

According to the stress cloud map, the maximum stress in composite conditions is $528.74 \mathrm{MPa}$, which meets our required strength requirements. When carrying out real vehicle test, it is necessary to pay attention to the force situation of weak parts.

\subsection{Modal analysis}

Modal analysis mainly has two types of free mode and constraint mode. Constraint modal analysis mainly considers the modal characteristics of components with peripheral boundary conditions, for automotive steering knuckle parts, the constraint conditions in its motion state are not easy to determine. It is very difficult to accurately simulate steering knuckle constraint mode on the finite element model. Free mode analysis can not only obtain the dynamic characteristics of the structure, such as natural frequency, vibration type, etc., but also has a certain guiding effect on the subsequent analysis of the stress response of the steering knuckle structure. Therefore, free modal analysis is used for steering knuckle in this paper [6].

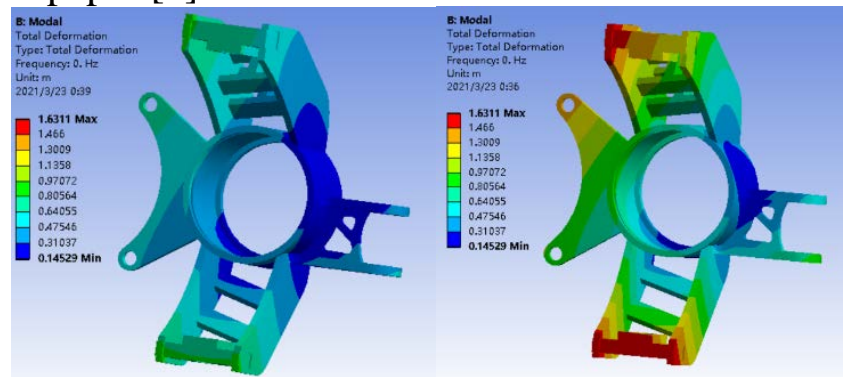

Figure 8. The seventh and eighth-order vibration patterns of the steering knuckle

With Ansys Workbench for modal analysis, in free modal analysis, the 1st-6th order is rigid modal and the inherent frequency is 0 . The mode above the 7th order is the flexible mode of the structure, and it is also the input caused by road unevenness, wheel rotation vibration, unbalanced vibration of the drive shaft and engine working vibration, and unevenness of the road surface .Typically around $20 \mathrm{~Hz}$, the maximum input frequency that may be caused by an imbalance in the wheel or transmission shaft is around $30 \mathrm{~Hz}$, while the input frequency caused by engine operation is about $200 \mathrm{~Hz}$, well 
below the seventh-order frequency of the steering knuckle $1631 \mathrm{~Hz}$, so the dynamic characteristics of the steering knuckle meet the requirements of use and do not trigger vehicle resonance.

\section{Conclusions}

Through finite element analysis of the steering knuckle, it can be seen that the safety margin of the steering knuckle is large, which can meet the strength requirements in various hazardous conditions, thus verifying that the design model is correct and feasible.

This article describes the product development and verification process of a new type of steering knuckle in more detail, and provides a systematic design process for the iterative process of the racing knuckle update. Figure 9 below shows the manufactured welded knuckle. However, in the process of simulation analysis, there are still some incomplete parts that need to be improved in the follow-up research.
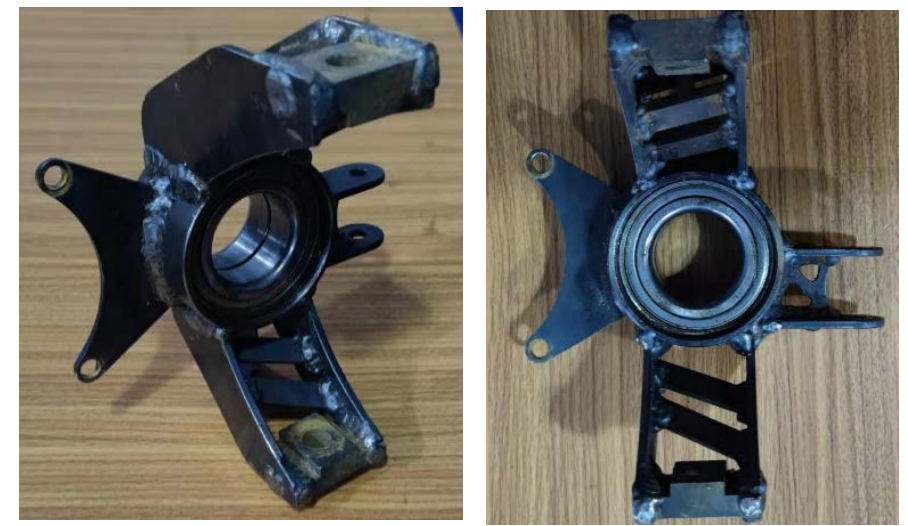

Figure 9. The manufactured welded knuckle

\section{Acknowledgments}

National innovation and entrepreneurship training program for college students S202010497160

\section{References}

[1] Huang Xiaoxuan. Modal study of a car steering knuckle based on finite meta-analysis, 2021, 37 (03): 45-47.

[2] Zhang Jingjing, Liu Yu, Chen Hai. Research on the performance simulation and testing of the pre-steering kinetics performance of an SUV based on ABAQUS, Automotive Practical Technology, 2021, 46 (03): 109-111.

[3] Jin Weigang, Shen Jian. Steering knuckle processing program optimization. Internal combustion engines and accessories, 2021 (02): 88-89.

[4] Zhou Yu, Hu Songhan, Fan Wei, Zhang Electric. Topologically Optimized Steering Arm Lightweight Design, Beijing Automotive, 2020 (06): 32-34.

[5] Liu Ying, Yan Yang, Chen Talent, Pang Qiu. Study on the optimization of steering knuckle structure taking into account load uncertainty, 2020, 37 (12): 1504-1509.

[6] Ma Xipei, Li Yawei, Fan Pingqing. Typical condition load-bearing analysis of steering knuckle based on system dynamics, combining machine tools and automated machining technology, 2020 (12): 39-42.

[7] Dong Guojie, Wang Wei, Du Fei, Lang Yuling. Virtual iterative method steering load-saving spectral extraction and verification of mechanical strength, 2020, 42 (06): 1323-1331. 